\title{
Long-range Single Baseline RTK GNSS Positioning for Land Cadastral Survey Mapping
}

\author{
Brian Bramanto ${ }^{1, *}$, Irwan Gumilar ${ }^{1}$, Muhammad Taufik ${ }^{1}$ and I Made D. A. Hermawan ${ }^{2}$ \\ ${ }^{1}$ Geodesy Research Group, Institut Teknologi Bandung, Indonesia \\ ${ }^{2}$ General Technology Indonesia
}

\begin{abstract}
In Indonesia, Global Navigation Satellite System (GNSS) has become one of the important tool in survey mapping, especially for cadastral purposes like land registration by using Real Time Kinematic (RTK) GNSS positioning method. The conventional RTK GNSS positioning method ensure high accuracy GNSS position solution (within several centimeters) for baseline less than 20 kilometers. The problems of resolving high accuracy position for a greater distance (more than 50 kilometers) becomes greater challenge. In longer baseline, atmospheric delays is a critical factor that influenced the positioning accuracy. In order to reduce the error, a modified LAMBDA ambiguity resolution, atmospheric correction and modified kalman filter were used in this research. Thus, this research aims to investigate the accuracy of estimated position and area in respect with short baseline RTK and differential GNSS position solution by using NAVCOM SF3040. The results indicate that the long-range single baseline RTK accuracy vary from several centimeters to decimeters due to unresolved biases.
\end{abstract}

\section{Introduction}

As a breakthrough technology in position determination, Global Navigation Satellite System (GNSS) has become one of the important tool in survey mapping. GNSS term includes e.g. the GPS (Global Positioning System), GLONASS (Globalnaya Navigazionnaya Sputnikovaya Sistema), Galileo, BeiDou and other satellite-based positioning system. In accordance with its rapid growth, there is such a huge increase interest in GNSS position determination, but not limited to, e.g. Automatic Vehicle Location (AVL) [1, 2], tracking system [3, 4], geodynamic monitoring [5-7], atmospheric monitoring [8, 9], hazard mitigation [10-12] and so on.

In Indonesia, GNSS is mostly used in surveying and mapping purposes, especially for cadastral purposes like land registration by using Real Time Kinematic (RTK) GNSS positioning method [13-15]. RTK GNSS ensure the high accuracy in point determination, however, in conventional RTK GNSS the high accuracy can only be obtained for baseline less than 20 kilometers [16]. For medium to long baseline RTK GNSS, the atmospheric bias is considered as the dominant factor which lead into unresolved ambiguity resolution. Consider GNSS signal travelling from a satellite to two receivers that are in a distant, the signal would be subjected to a different atmospheric effects. Several approaches have been proposed to mitigate the atmospheric bias [17-18]. Network RTK is also considered to mitigate the atmospheric bias [19].

It has been found that atmospheric bias affect more error in vertical component rather than in horizontal component which up to several decimeters in RTK GNSS positioning [20, 21]. Fig. 1 shows the comparison of code absolute method positioning error using corrected pseudorange and uncorrected pseudorange. It could be seen that the deviation could vary up to 20 meters in vertical component. The corrected terms indicate the used of troposphere and ionosphere model.

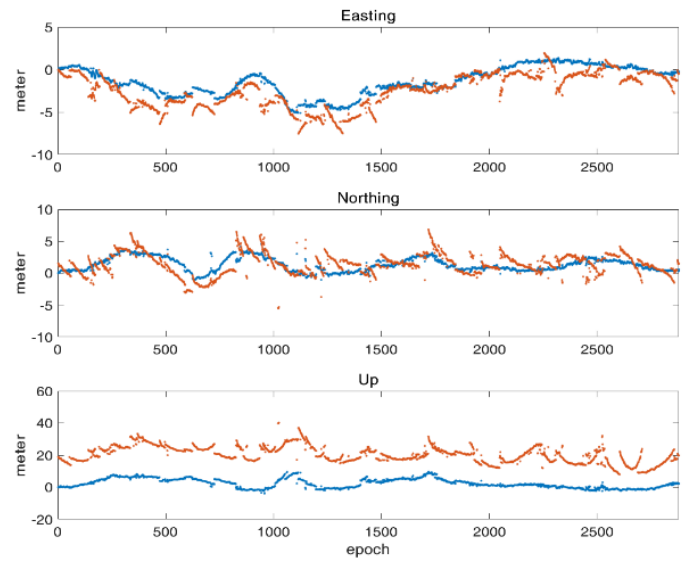

Fig. 1. Error position in absolute positioning method. Red dots indicate when no atmospheric correction was applied on the data, while blue dots indicate when atmospheric correction was applied on the data

Thus, several researches has stated that orbital error [22] and satellite clock error [23] also indicate as the problems in GNSS-based positioning system. The orbital trajectory of GNSS satellites disturbed by surrounding environments e.g. the Earth's gravity, the attraction of the

\footnotetext{
* Corresponding author: brian@ fitb.itb.ac.id
} 
sun and the moon and as well as solar radiation, while the satellite clocks are subject to relativistic effects. The GNSS satellite clock tends to run faster than the clocks in the receivers.

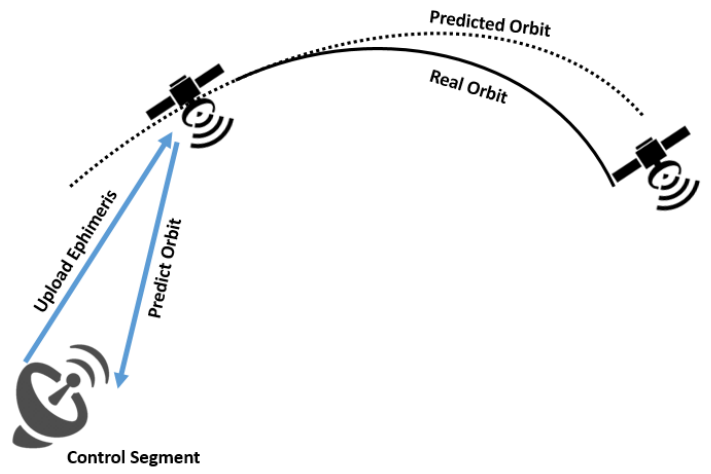

Fig. 2. GNSS orbital satellite error

In a relatively short baseline, the double difference (DD) observations could reduce and eliminate both of orbital and clock satellite error, however, for a far baseline orbital and clock satellite error still contained on the data observation. The connection between baseline length, observing time and rms accuracy were summarize in Fig. 3.

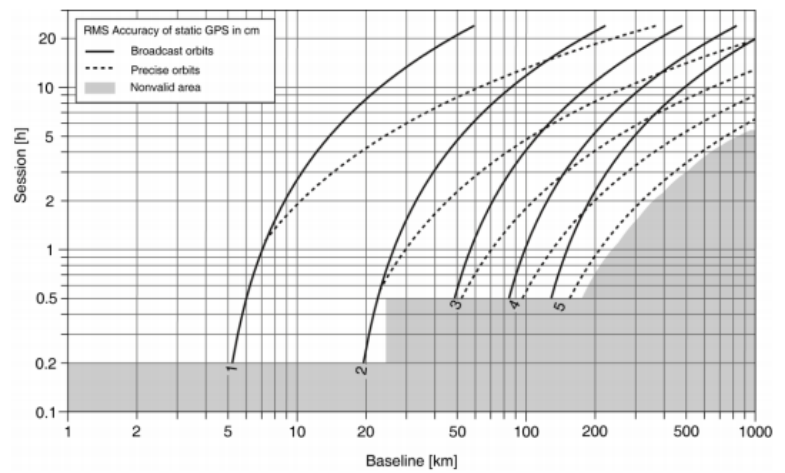

Fig. 3. Accuracy of GNSS static in $\mathrm{cm}$ and its correlation with the baseline length and observing time when using broadcast orbit and precise orbit [22]

In this research, a relatively new algorithm [23] was used to enhance the RTK GNSS accuracy in a long baseline for land cadastral survey mapping. This method used a modified LAMBDA method which can be separated into several aspects e.g. modified functional model to estimate the atmospheric bias, the usage of precise orbit correction from WADGPS, a modified Kalman filter and a partial search and ambiguity fixing strategies.

\section{Data and Basic Concept}

\subsection{Data}

Base station was established at the rooftop building. Bandung, Indonesia, while the land cadastral survey mapping were simulated on three land parcels in Pamengpeuk, Indonesia which located for about 85 kilometers away from the base station and has significant height differences for about 800 meters. Eight benchmarks were also used to assess the performance of the algorithm.

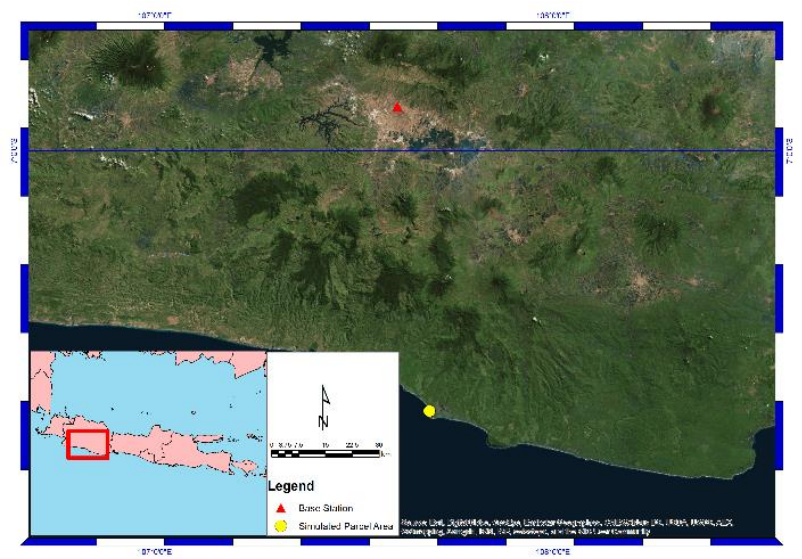

Fig. 3. The location of base station (red triangle) and simulated parcel area (yellow dot)

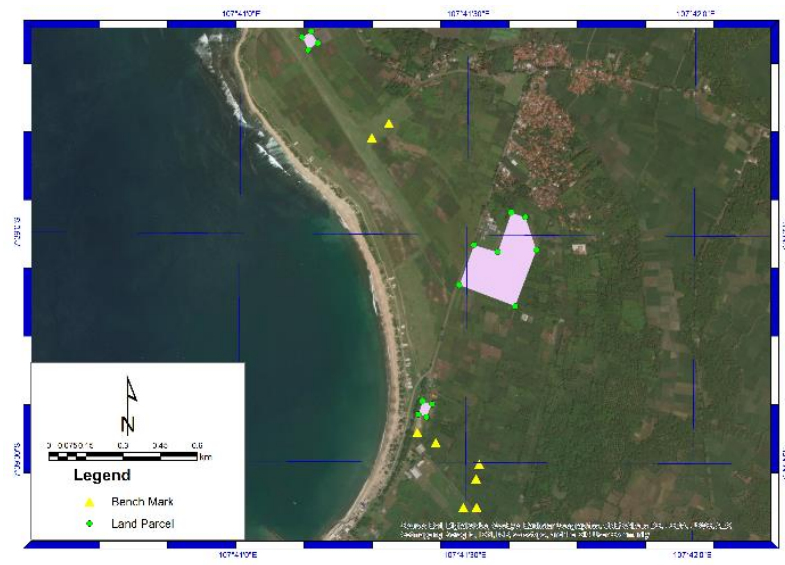

Fig. 4. The location of land parcels (green dot) and benchmark (yellow triangle)

Fig. 5. Shows the research methodology. In general, to assess the performance of the algorithm, the long-range RTK coordinate results were then compared with a priori coordinates. The term a priori coordinate refers to reference coordinate based on static differential observation method or shorter baseline RTK GNSS method. 


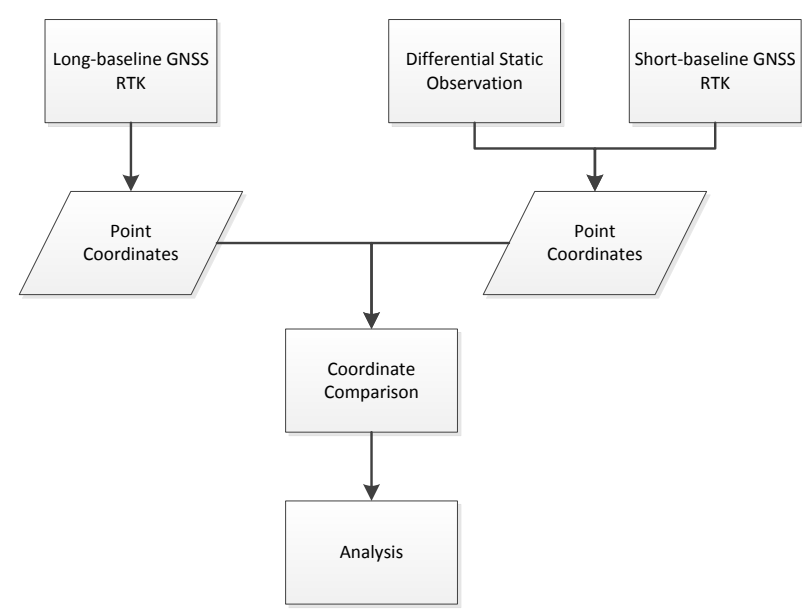

Fig. 5. Research methodology used in this research

\subsection{Basic Concept}

This section describe the general concept in RTK GNSS method and the Kalman filter design to enhance the accuracy of RTK GNSS in long-range baseline.

\subsubsection{Observation Models in RTK}

The observation model for code and carrier phase measurement are described as follows:

$$
\begin{aligned}
& P(L i)=\rho+d_{\text {orb }}+d_{\text {trop }}+d_{\text {ion }_{i}}+c(d t-d T)+ \\
& M P i+\varepsilon_{i} \\
& \Phi(L i)=\rho+d_{\text {orb }}+d_{\text {trop }}-d_{\text {ion }}+c(d t-d T)+ \\
& \lambda_{i} N_{i}+M \Phi i+\varepsilon_{i}
\end{aligned}
$$

where:

$P(L i) \quad$ is the measured pseudorange on $L i$ frequency

$$
(i=1,2)
$$

$\Phi(L i) \quad$ is the measured carrier phase on $L i$ frequency

$\rho \quad$ is the true geometric range

$d_{\text {orb }} \quad$ is the satellite orbital error

$d_{\text {trop }} \quad$ is the tropospheric error

$d_{\text {ion }} \quad$ is the ionospheric error

$c \quad$ is the speed of light

$d t \quad$ is the satellite clock error

$d T \quad$ is the receiver clock error

$M P i \quad$ is the multipath effect on measured code

$M \Phi i \quad$ is the multipath effect on measured phase $\varepsilon_{i} \quad$ is the noise

DD then performed to eliminate the orbital error, clock error and atmospheric error in short baseline. The DD $(\Delta \nabla)$ observation model for code and carrier phase measurement can be described as follows:

$$
\begin{aligned}
& \Delta \nabla \Phi(L i)_{A B}^{j k}=\Delta \rho_{A B}^{j k}+\lambda_{i} N_{i}^{j k}+M \Phi i_{A B}^{j k}+\varepsilon_{i}^{j k}{ }_{A B}^{j k} \\
& \Delta \nabla P(L i)_{A B}^{j k}=\Delta \rho_{A B}^{j k}+M P i_{A B}^{j k}+\varepsilon_{i}^{j k}
\end{aligned}
$$

Linearization of the DD observation then can be represented as follows:

$V=A X-L$

where $\mathrm{V}$ is the residual matrix, $A$ is the design matrix. $L$ is the observation data and $X$ is the estimated parameters containing three baseline components and ambiguities. In a longer baseline the estimated parameters including residual ionospheric bias and residual tropospheric bias.

\subsubsection{General Kalman Filter System Design}

Kalman filter predicts the a priori parameters using the recent estimate of the observation data. The prediction is based on some assumed model for how the parameters changes in time $[24,25]$. The dynamic model on Kalman filter can be represented as follows:

$X_{K+1}=\Phi_{k} X_{k}+w_{k}$

which then continued along with measurement model,

$L_{k}=A_{k} X_{k}+v_{k}$

where:

$\Phi_{k} \quad$ is the transition matrix $(k=$ epoch $)$

$w \quad$ is the noise from the dynamic model

$v \quad$ is the noise from the observation data

Kalman filter also applied recursive least square which then can be defined into two main parts as follows [26]:

- Observation model

$K_{k}=P_{k}^{-} A_{k}^{T}\left(A_{k} P_{k}^{-} A+R_{k}\right)^{-1}$

$X_{k}=X_{k}^{-}+K_{k}\left(L_{k}-A_{k} X_{k}^{-}\right)$

$P_{k}=\left(I-K_{k} A_{k}\right) P_{k}^{-}$

- Dynamic model

$X_{k+1}^{-}=\Phi_{k} X_{k}$

$P_{k+1}^{-}=\Phi_{k} P_{k} \Phi_{k}^{T}+Q_{k}$

where:

$K \quad$ is the gain matrix

$P \quad$ is the covariance matrix observation model

I is the identity matrix

- $\quad$ is the prediction from previous epoch

$Q \quad$ is the covariance matrix for dynamic model

$R \quad$ is the weighting matrix

The estimated parameter is summarized in Table 1.

Table 1. Parameter estimated in Kalman filter. Superscript $\left(^{*}\right)$ indicates the optional parameter while $\mathrm{N}$ is the number of satellites used 


\begin{tabular}{|l|c|}
\hline $\begin{array}{l}\text { Parameter in Kalman } \\
\text { Filter }\end{array}$ & Dimension \\
\hline Position XYZ & 3 \\
\hline Velocity XYZ* & 3 \\
\hline Acceleration XYZ* & 3 \\
\hline Residual troposphere* & 1 \\
\hline Residual DD ionosphere* & $\mathrm{N}-1$ \\
\hline L1 DD ambiguity* & $\mathrm{N}-1$ \\
\hline L2 DD ambiguity* & $\mathrm{N}-1$ \\
\hline
\end{tabular}

\subsubsection{Precise Satellite Ephemeris}

As stated on the introduction, precise satellite ephemeris is needed in GNSS-based point positioning for a longer baseline. In static differential method, the precise satellite ephemeris can be easily obtained two weeks after the observation is done. However, in conventional RTK such a precise ephemeris cannot be obtained. Several researches indicate that the satellite's position error might vary up to 5 meters $[22,27]$. The satellite's position error is generally the biggest error source after atmospheric bias is estimated in Kalman filter for long-range RTK.

Thus several GNSS industries have developed their own system to accommodate the use of precise satellite ephemeris. John Deere, as one of the GNSS industry has developed the StarFire ${ }^{\mathrm{TM}}$ system which transmits the needed data correction in near real-time using satellites communication.

\subsubsection{Ambiguity Resolution}

The ambiguities are considered as constant. However, due to remaining tropospheric and ionospheric biases, the ambiguities can be modeled as a random walk with very small dynamic noise, such as 0.001 cycle. Thus, the ambiguities are modeled as constants once the ambiguities are fixed. The use of these small dynamic noise is useful in resolving the ambiguities in several condition, such as bad site condition, excessive multipath, or the movement of receiver from a severe shading surrounding to the open sky surrounding.

In a longer baseline, the ambiguities are resolved improperly due to significant bias. [7] implemented the modified partial search technique to fixing the ambiguities. The ambiguity for the L1/L2 signal and its variance were first converted into L1/Wide Lane (WL) as described in following vector equation:

$$
\left(\begin{array}{c}
\widehat{L_{1}} \\
\widehat{W L}
\end{array}\right)=\left(\begin{array}{cc}
1 & 0 \\
1 & -1
\end{array}\right)\left(\begin{array}{c}
\widehat{L_{1}} \\
\widehat{L_{2}}
\end{array}\right)
$$

$$
Q_{\widehat{L_{1}}, \widehat{W L}}=\left(\begin{array}{cc}
1 & 0 \\
1 & -1
\end{array}\right)\left(\begin{array}{cc}
Q_{\overparen{L_{1}}} & Q_{\widehat{L_{1}}, \widehat{L_{2}}} \\
Q_{\widehat{L_{1}}, \widehat{L_{2}}} & Q_{\widehat{L_{2}}}
\end{array}\right)\left(\begin{array}{cc}
1 & 1 \\
0 & -1
\end{array}\right)
$$

The used of WL is important due to its wavelength characteristic. With $0.86 \mathrm{~cm}$ wavelength, WL's ambiguities are easy to resolve. If the ambiguities are resolved, the original L1 and L2 ambiguities and the variance-covariance in the Kalman filter can be recovered as follows:

$$
\begin{aligned}
\left(\begin{array}{c}
\widehat{N_{1}} \\
\widehat{N_{2}}
\end{array}\right)=\left(\begin{array}{cc}
1 & 0 \\
1 & -1
\end{array}\right)\left(\begin{array}{c}
\widehat{X_{1}} \\
\widehat{X_{W L}}
\end{array}\right) \\
Q_{\widehat{N_{1}}, \widehat{N_{2}}}=\left(\begin{array}{cc}
1 & 0 \\
1 & -1
\end{array}\right)\left(\begin{array}{cc}
Q_{\widehat{N_{1}}} & Q_{\widehat{N_{1}}, \widehat{N_{W L}}} \\
Q_{\widehat{N_{1}}, \widehat{N_{W L}}} & Q_{\widehat{N_{W L}}}
\end{array}\right)\left(\begin{array}{cc}
1 & 1 \\
0 & -1
\end{array}\right)
\end{aligned}
$$

\section{Result and Discussion}

Over 1840 epoch were collected within 23 point observations. Only resolved ambiguities data showed and considered in further analysis. Coordinates derived from differential static method were considered as reference coordinates in bench mark point, while coordinates derived from short baseline GNSS RTK method were used as reference coordinates in land parcel point. Short baseline GNSS RTK (under $3 \mathrm{~km}$ ) was considered because in shorter baseline and in the open-sky condition (Fig. 6) the biases were assumed reduced or eliminated [28].

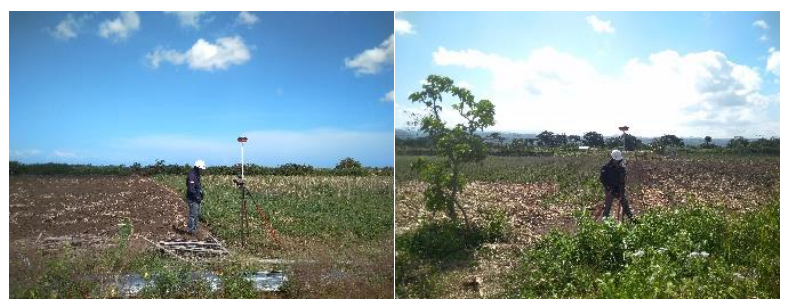

Fig. 6. Condition over the simulated area

\subsection{Accuracy and Precision}

\subsubsection{Horizontal Accuracy}

Fig 7. shows the overall accuracy for benchmark point, while Fig 8. shows the overall accuracy for land parcel point. The accuracy of long-baseline GNSS RTK in benchmark points were within $3 \mathrm{~cm}$ and only 1 point was slightly worse than the other, however still within RTK accuracy.

The accuracy of long-baseline GNSS RTK in land parcel points were within $12 \mathrm{~cm}$. It could be seen that there was a systematic compared with those for benchmark point. As mentioned before, coordinate estimated from short baseline RTK GNSS used to assess the accuracy of long baseline RTK GNSS in land parcel points. To evaluate the consistency of the used reference coordinate on all off the observation method, benchmark points were also observed using short baseline RTK GNSS. 


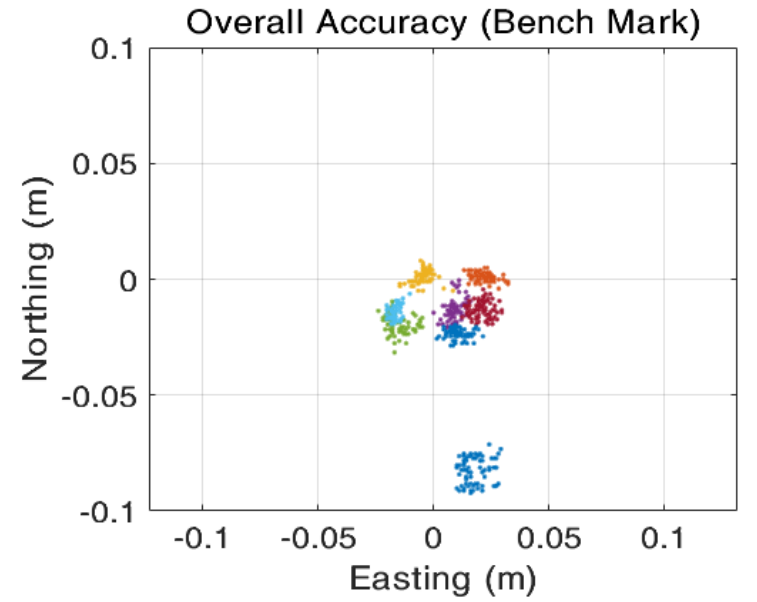

Fig. 7. Overall accuracy of long baseline GNSS RTK for benchmark points

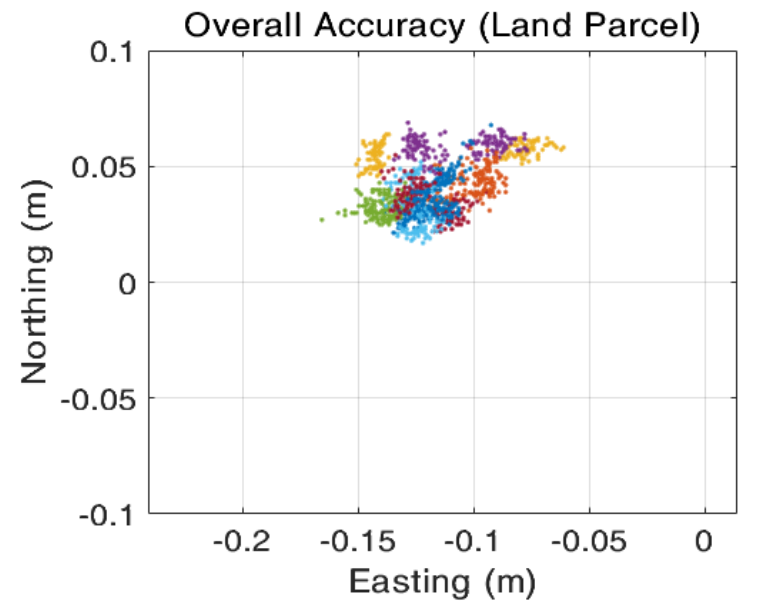

Fig. 8. Overall accuracy of long baseline GNSS RTK for land parcel points

Fig. 9. shows the overall accuracy for both short and long baseline GNSS RTK. It could be seen that there was a shift tendencies to South-East. It indicates that the system coordinate might be different.

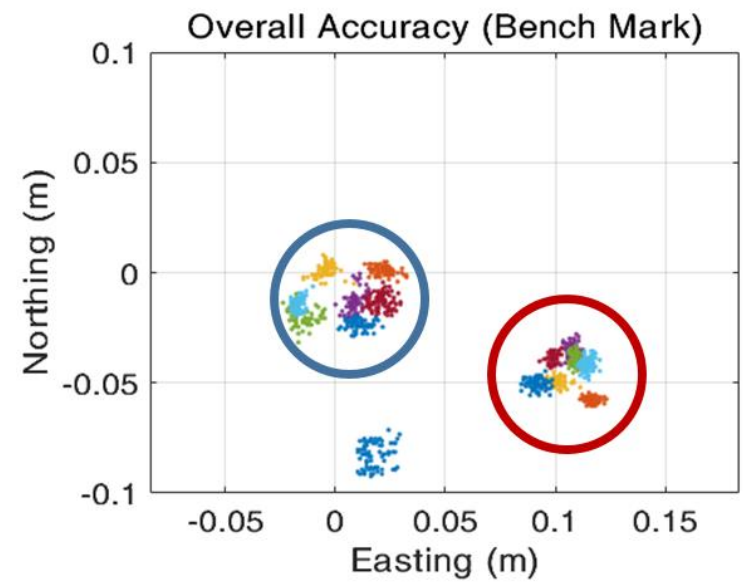

Fig. 9. Overall accuracy of short baseline (Red Circle) and long baseline (Blue Circle) GNSS RTK for benchmark points
Fig 10. shows the overall vertical accuracy for benchmark point, while Fig 11. shows the overall vertical accuracy for land parcel point. The accuracy of long-baseline GNSS RTK in benchmark points were within $15 \mathrm{~cm}$ and the accuracy of long-baseline GNSS RTK in land parcel points were vary from $-20 \mathrm{~cm}$ to $15 \mathrm{~cm}$. There is one point that indicates the unresolved bias. A linear trend of the up component is found on that point, there is also deviation in horizontal component as shown on Fig. 7. Further analysis is needed to explain this phenomenon.

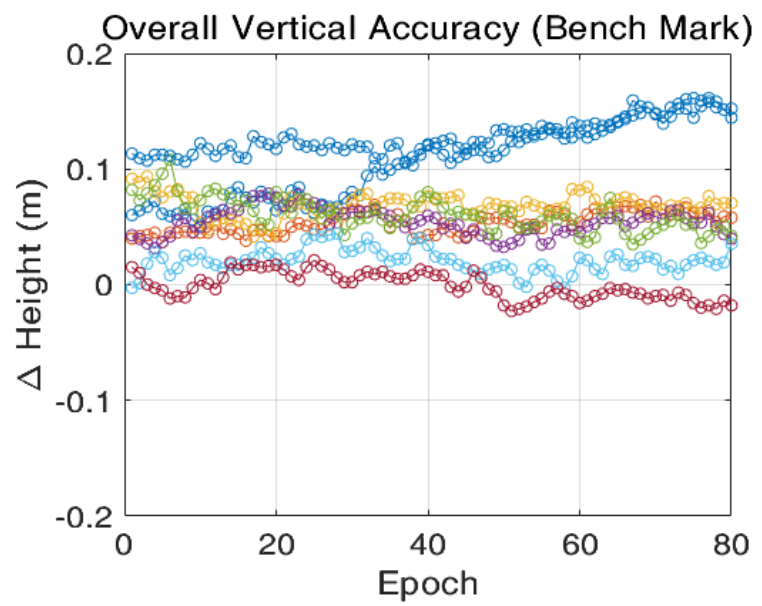

Fig. 10. Overall vertical accuracy of long baseline GNSS RTK for benchmark points

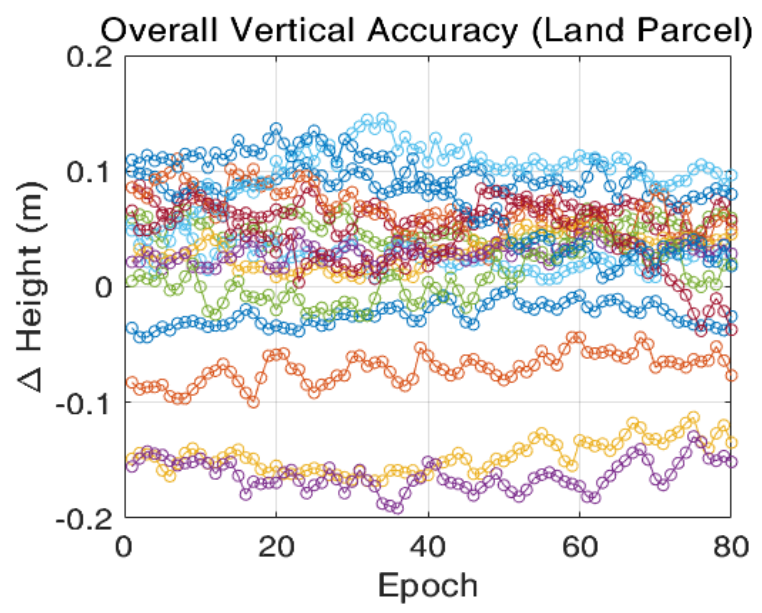

Fig. 11. Overall vertical accuracy of long baseline GNSS RTK for land parcel points

\subsubsection{Vertical Accuracy}




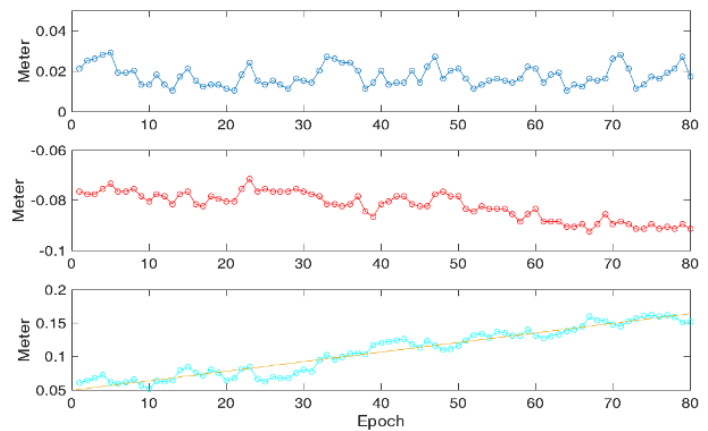

Fig. 12. Selected timeseries of Blue dot, red dot and cyan dot refer to easting, northing and up component respectively. Yellow lines indicates the linear trend of up component.

\subsubsection{Overall Precision}

Table 1. shows the overall precision for long baseline GNSS RTK. Precision indicated the repeatability of the estimated coordinate. Over $90 \%$ of estimated coordinate met the 95\% of confidence interval as shown on Fig.13. This indicate that this algorithm is reliable to used.

Table 1. Overall precision for long baseline GNSS RTK

\begin{tabular}{|c|c|c|c|}
\hline Easting (m) & Northing (m) & Up (m) & Note \\
\hline 0.0055 & 0.0050 & 0.0345 & BM \\
\hline 0.0020 & 0.0044 & 0.0093 & BM \\
\hline 0.0028 & 0.0034 & 0.0102 & BM \\
\hline 0.0045 & 0.0031 & 0.0117 & BM \\
\hline 0.0044 & 0.0048 & 0.0143 & BM \\
\hline 0.0029 & 0.0018 & 0.0106 & BM \\
\hline 0.0036 & 0.0040 & 0.0117 & BM \\
\hline 0.0021 & 0.0040 & 0.0142 & BM \\
\hline 0.0026 & 0.0042 & 0.0098 & Land Parcel \\
\hline 0.0057 & 0.0083 & 0.0130 & Land Parcel \\
\hline 0.0028 & 0.0064 & 0.0138 & Land Parcel \\
\hline 0.0031 & 0.0064 & 0.0129 & Land Parcel \\
\hline 0.0042 & 0.0066 & 0.0098 & Land Parcel \\
\hline 0.0042 & 0.0056 & 0.0114 & Land Parcel \\
\hline 0.0039 & 0.0044 & 0.0110 & Land Parcel \\
\hline & & & \\
\hline
\end{tabular}

\begin{tabular}{|l|l|l|l|}
\hline 0.0047 & 0.0046 & 0.0088 & Land Parcel \\
\hline 0.0062 & 0.0036 & 0.0195 & Land Parcel \\
\hline 0.0049 & 0.0030 & 0.0135 & Land Parcel \\
\hline 0.0047 & 0.0050 & 0.0078 & Land Parcel \\
\hline 0.0035 & 0.0058 & 0.0203 & Land Parcel \\
\hline 0.0054 & 0.0057 & 0.0240 & Land Parcel \\
\hline 0.0053 & 0.0050 & 0.0294 & Land Parcel \\
\hline 0.0037 & 0.0057 & 0.0408 & Land Parcel \\
\hline
\end{tabular}

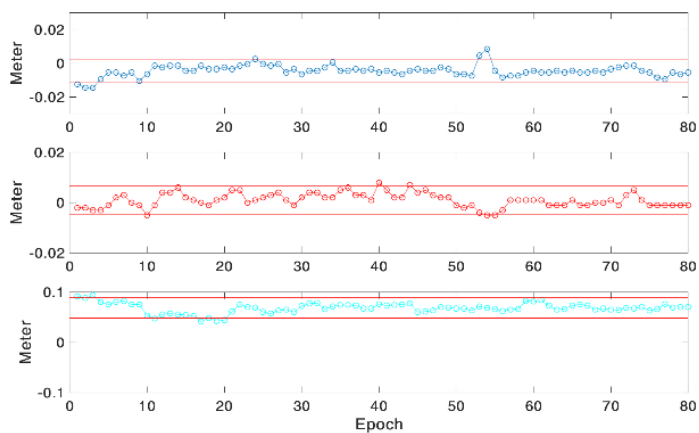

Fig. 13. Selected timeseries of estimated coordinate for long baseline GNSS RTK. Blue dot, red dot and cyan dot refer to easting, northing and up component respectively. Red lines indicates the $95 \%$ of confident interval.

\subsubsection{Area Estimation}

Government policy about land and building tax in Indonesia indicates that the errors tolerances is about $10 \%$. Table.2. shows the differences in calculated area with the reference area, there is no significant differences between reference and calculated area. The deviation in under $0.05 \%$ for each area. This result indicates that the long baseline GNSS RTK algorithm can be used for land parcel mapping.

Table 2. Area differences

\begin{tabular}{|l|l|l|l|c|}
\hline ID & $\begin{array}{l}\text { Reference } \\
\text { Area }\end{array}$ & $\begin{array}{l}\text { observed } \\
\text { Area }\end{array}$ & $\begin{array}{l}\text { Area } \\
\text { Differences }\end{array}$ & \% \\
\hline 1 & 2490.9708 & 2490.1062 & 0.8646 & $0.03 \%$ \\
\hline 2 & 63787.5385 & 63779.2118 & 8.3267 & $0.01 \%$ \\
\hline 3 & 2111.4568 & 2111.1478 & 0.309 & $0.01 \%$ \\
\hline
\end{tabular}




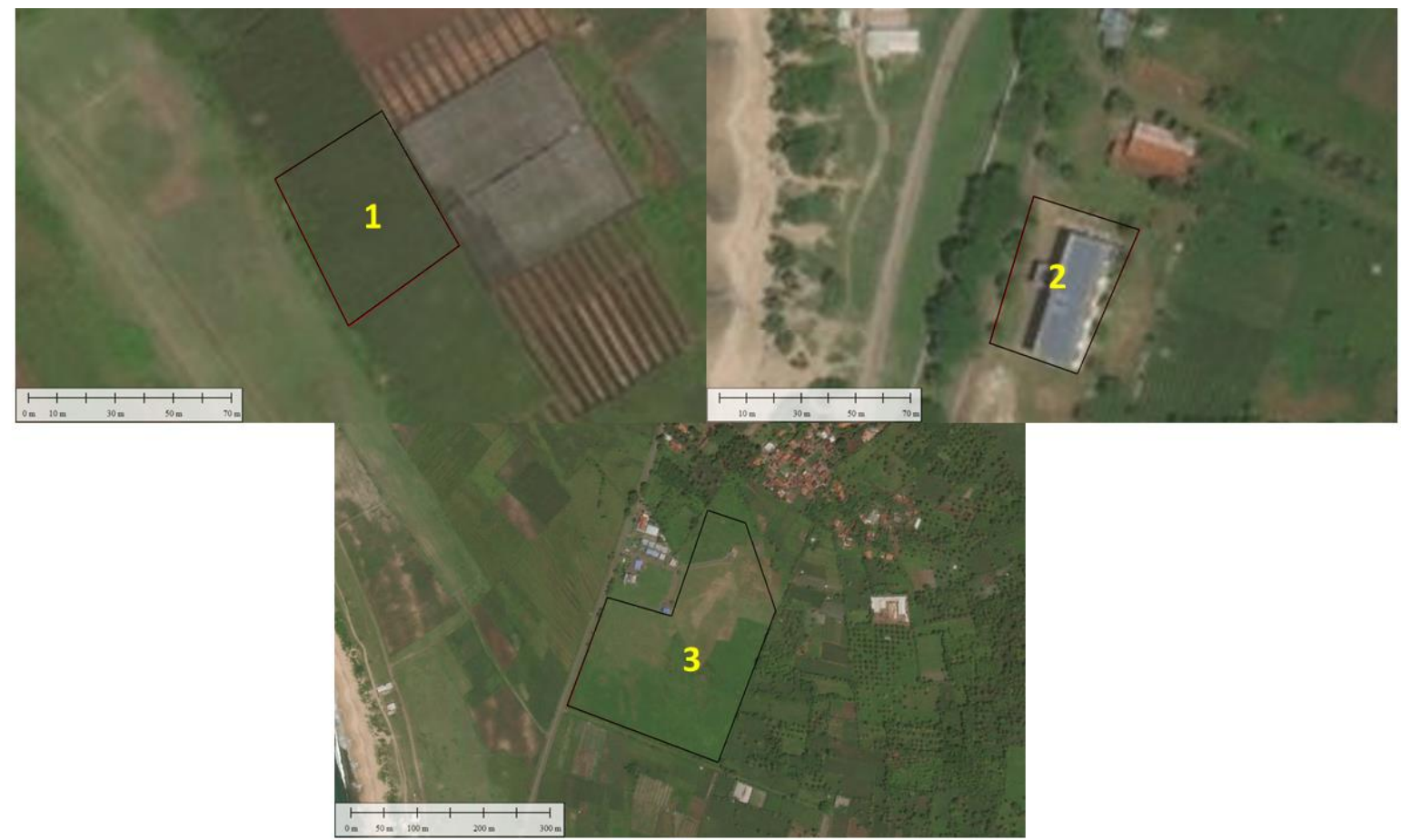

Fig. 13. Formed area over simulated area. Black lines indicate the reference area while red lines indicate the observed area.

\section{Conclusion}

The algorithm gives significant improved in long-range single baseline GNSS RTK for up to $90 \mathrm{~km}$. The accuracy vary from several centimeters to decimeters due to unresolved biases. For land cadastral purposes, the algorithm can be used as one of the method, the observed area shows no significant difference compared with the reference area.

\section{References}

1. Qing-Jie Kong, Zhipeng Li, Yukai Chen, Yuncai Liu. "An Approach to Urban Traffic State Estimation by Fusing Multisource Information" In IEEE Transactions on Intelligent Transportation System 10.3 (2009) pp. 499-511'

2. E. Mazloumi, G. Currie, and G. Rose. "Using GPS data to gain insight into public transport travel time variability". In: Journal of Transportation Engineering 136.7 (2010), pp. 623-631.

3. Sathe Pooja "Vehicle Tracking System using GPS" In: International Journal of Science and Research (IJSR), India Online ISSN: 2319-7064 (2013)

4. Matej Supej and Ivan Čuk "Comparison of Global Navigation Satellite System Devices on Speed Tracking in Road (Tran)SPORT Applications" In: Sensor 2014, 14 23490-23509 (2014)

5. M. Ben Rabha, M.F. Boujmil, M. Saadoun, B. Bessaïs, Eur. Phys. J. Appl. Phys. (to be published)
6. Luigi T. De Luca, Propulsion physics (EDP Sciences, Les Ulis, 2009)

7. F. De Lillo, F. Cecconi, G. Lacorata, A. Vulpiani, EPL, 84 (2008)

8. Brian Bramanto, Irwan Gumilar, Teguh P. Sidiq, Wedyanto Kuntjoro, Daniel A. Tampubolon "Sensing of the atmospheric variation using low cost GNSS receiver" IOP Conference Series Earth and Environmental Science 149(1):012073 (2018)

9. P. Xia, C. Cai, Z. Liu "GNSS troposphere tomography based on two-step reconstruction using GPS observation adn COSMIC Profiles" Ann. Geophys., 31, (2013) pp 1805-1815

10. K. M. Kafi, M. B. A. Gibril "GPS Application in Disaster Management: A Review" Asian Journal of Applied Sciences, 4:01 (2016)

11. Y. Lin, C. Huang, S. Chen, D. Doong, C. C. Kao "Development of a GNSS Buoy for Monitoring Water Surface Elevations in Estuaries and Coastal Areas" Sensors 17(1) (2017) pp 1-19

12. C. Rizos, S. Han, L. Ge, H. Chen, Y. Hatanaka, K. Abe "Low-cost densification of permanent GPS networks for natural hazard mitigation: First tests on GSI's GEONET network" Earth Planet Space, 51 (2000) pp 867-871

13. A. Priti, N. Arslan, B. Deveci, O. Aydin, H. Erkaya, R. G. Hosbas "Real-time kinematic GPS for cadastral surveying" Survey Review vol. 41(314) (2009)

14. Hasanuddin Z. Abidin, Tony S. Haroen, F. H. Adiyanto, Heri Andreas, Irwan Gumilar, Imam Mudita, I. Soemarto "On the establishment and implementation of GPS CORS for cadastral 
surveying and mapping in Indonesia" Survey Review 47(340) (2015) pp 61-70

15. H. Z. Abidin, T. S. Haroen, I. Mudita, F. H. Adiyanto "Implementation of GPS CORS for Cadastral Survey and Mapping in Indonesia: Status, Constraints and Opportunities" FIG Working Week 2012 (2012)

16. Yanmin Feng and Jinling Wang "GPS RTK Performance Characteristics and Analysis" Journal of Global Positioning Systems 2008 Vol. 7, No. 1 : 1-8 (2008)

17. P. Wielgosz, I. Kashani and D. Grejner-Brzezinska "Analysis of long-range network RTK during a severe ionospheric storm" In: J. Geod. 2005:79 (2005) pp 524-531

18. Y.W. Ahn, D. Kim, P. Dare and J. Park "Estimation of troposphere decorrelation using the combined zenith-dependent parameter" In: Proceedings of the ION GNSS 2008; Savannah, GA, USA. (2008) pp. 261-270.

19. S. Han and C. Rizos "GPS network design and error mitigation for real-time continuous array monitoring system” In: Proceedings of the ION GPS 1996 (1996) pp. 1827-1836.

20. H. Guo, H. He, J. Li and A. Wang "Estimation and mitigation of the main errors for centimetre-level COMPASS RTK solutions over medium-long baselines" In: J. Navig. 2011;64 (2011) pp 113-126

21. Y. Feng and B. Li "Wide area real time kinematic decimetre positioning with multiple carrier GNSS signals" In: Sci. China Ser. D. 2010;53 (2010) pp 731-740

22. Pasi Häkli, Hannu Koivula and Jyrki Puupponen "Assessment of Practical 3-D Geodetic Accuracy for Static GPS Surveying” In: FIG Working Week 2008 (2008)

23. Bharati Bidikar, Gottapu Sasibhushana Rao, Laveti Ganesh and MNVS Santosh Kumar "Satellite Clock Error and Orbital Solution Error Estimation for Precise Navigation Applications" In: Positioning, 2014, 5 (2014) pp 22-26

24. B. Witchayangkoon "Elements of GPS Precise Point Positioning" In: Doctoral Disertation (2000)

25. M. S. Grewal and A. P. Andrews "Kalman Filtering Theory and Practices Using MATLAB” (2008)

26. R. G Brown and P. Y. C. Hwang "Introduction to Random Signals and Applied Kalman Filtering" (2012)

27. Janusz Zielinski and Ryszard Zdunek "GPS orbit accuracy and its influence on large network solutions" In: Advances in Space Research 23(4):665-665 DOI: 10.1016/S0273-1177(99)00149$0,(1999)$

28. B. Bramanto, I. Gumilar, H. Z. Abidin, K. Prijatna, F. S. Adi “Assessment of the BeiDou Data Quality and the Positioning Performance: A Perspective from Bandung, Indonesia" Journal of Aeronautics, Astronautics and Aviation, Series A 49(2) (2017) 